\title{
Development and Assessment of a Prenatal Cytomegalovirus (CMV) Educational Survey: Implementation and Impact in a Metropolitan University-Based Clinic
}

International Journal of Women's Health

\author{
Malinda R Schaefer' \\ Jessica Holttum (D) \\ Megan Olson' \\ Danielle Westenberg' \\ Nathan Rubin (iD ${ }^{2}$ \\ Mark R Schleiss (iD ${ }^{3}$ \\ Jessica Nyholm' \\ 'Department of Obstetrics, Gynecology \\ \& Women's Health, University of \\ Minnesota Medical School, Minneapolis, \\ MN 55455, USA; ${ }^{2}$ Biostatistics Core, \\ Masonic Cancer Center, University of \\ Minnesota Medical School, Minneapolis, \\ MN 55455, USA; ${ }^{3}$ Department of \\ Pediatrics, Division of Pediatric Infectious \\ Diseases and Immunology, University of \\ Minnesota Medical School, Minneapolis, \\ MN 55455, USA
}

Correspondence: Malinda R Schaefer Email schaefer.malinda@gmail.com

\begin{abstract}
Purpose: Congenital CMV infection can result in serious sequelae in the newborn. The goal of this study was to assess pregnant women's knowledge and understanding of CMV infection during pregnancy and develop an educational tool about CMV infection to be utilized during prenatal care.

Materials and Methods: This is a prospective intervention study that assessed pregnant women's knowledge before and after receiving an educational handout about CMV infection in pregnancy and the perceived value of this education. Pre- and post-education questionnaires were utilized to assess knowledge. The pre-education questionnaire and CMV educational handout were given at the same clinic visit. The educational handout was given after the pre-education questionnaire had been completed. The post-education questionnaire was given at the next scheduled prenatal clinic appointment and included questions regarding the level of satisfaction with the education and the perceived value of the information. Pregnant women less than 34 weeks of gestation were eligible.
\end{abstract}

Results: A total of 263 women were enrolled, 263 completed the pre-CMV educational questionnaire and 215 women completed both questionnaires. Some women only partially completed the questionnaires and those partial responses have been included. Prior to education, $33 \%$ (85/261) of participants had heard of CMV. This increased to $75 \%$ (160/214) after education. Participants scored each of the recommended hygiene practices between 1 and 5 (5 is the most acceptable) and each recommended hygiene practice received an average score between 3.8 and $5.74 \%(134 / 180)$ of participants reported increasing their hygienic practices after education. $96 \%(180 / 188)$ of participants indicated they were satisifed to have received the education. $98 \%$ (187/190) thought more women should receive this education during prenatal care.

Conclusion: Pregnant women viewed education about CMV favorably and increased the frequency of recommended hygiene practices. Introducing an educational handout to routine prenatal care may be beneficial in increasing awareness of CMV infection in pregnancy.

Keywords: congenital CMV, CMV vaccine, CMV pregnancy, CMV education

\section{Introduction}

Congenital cytomegalovirus (cCMV) is a significant cause of fetal and neonatal morbidity and mortality, leading to long-term sensorineural hearing loss, vision impairment, cognitive impairment and neurodevelopmental delay. ${ }^{1,2}$ The burden due to cCMV has been estimated to exceed that of other congenital conditions, 
including Down syndrome, fetal alcohol syndrome and spina bifida. ${ }^{3,4}$ However, despite this, the majority of reproductive-age women have never heard of $\mathrm{CCMV}^{5-8}$ and it is not routinely discussed during prenatal care. ${ }^{9-11}$ The estimated birth prevalence rate in industrialized countries is $0.6-0.7 \% .^{12,13}$ Often the diagnosis of cCMV is delayed because the majority of affected neonates are asymptomatic at birth $(\sim 87 \%) .{ }^{12}$ Of these asymptomatic neonates, $13.5 \%$ will develop permanent sequelae. ${ }^{12}$ However, in neonates with symptoms at birth, 40-58\% will have permanent sequelae. ${ }^{12}$

Congenital CMV infection can occur after maternal primary CMV infection or recurrent maternal infection. In the United States, it is estimated $30-50 \%$ of women under the age of 45 are CMV seronegative and at risk for primary CMV infection. ${ }^{3}$ Pregnancies in which maternal primary CMV infection occurs have the greatest risk of cCMV infection. ${ }^{14}$ These pregnancies have an estimated congenital infection rate of $32 \%$ compared to a $1.4 \%$ congenital infection rate during recurrent maternal infection. ${ }^{13}$ Despite the lower rate of congenital infection during recurrent infection, an estimated $75 \%$ of congenital CMV infections occur among women who were CMV seropositive prior to pregnancy. ${ }^{3,15,16}$

Cytomegalovirus is present in saliva, urine, semen, cervical/vaginal secretions and breast milk and is transmitted through direct contact with these bodily fluids. Young children, particularly children enrolled in daycare, have been identified as a significant source of CMV. It is estimated that $15-70 \%$ of children enrolled in daycare will become infected with CMV and can shed virus in their urine and saliva for up to 42 months. ${ }^{17,18}$ Currently, there is no licensed vaccine to prevent CMV infection and there are no proven medical therapies for either prevention of fetal transmission and/or treatment of acquired CMV infection in utero. The American College of Obstetrician and Gynecologists, the Society for Maternal and Fetal Medicine and the Centers for Disease Control and Prevention (CDC) recommend increased hand hygiene practices for prevention of CMV infection (Box 1) and recent studies have shown these recommendations are well-received by women. ${ }^{3,19,20}$ Additionally, there is a growing body of evidence showing that education about CMV infection, congenital CMV and the recommended increased hand hygiene practices are accepted by women and can decrease the CMV seroconversion rate during pregnancy. ${ }^{21-25}$ Furthermore, there is a growing consensus among clinicians that CMV education should
Box I Recommended Hygiene Practices to Prevent CMV Infection

- Wash your hands with soap and water for 15-20 seconds after changing diapers, feeding young children, or wiping a young child's nose

- Do not share food, drink or eating utensils with young children

- Do not put your child's pacifier in your mouth

- Do not share a toothbrush with your child

- Avoid contact with saliva when kissing a child

- Clean toys, countertops and other surfaces that come into contact with children's urine or saliva

Note: Recommendations from these studies. ${ }^{8,28,29}$

be provided during routine prenatal care $^{26}$ and several states have passed legislation requiring CMV education for women of child-bearing age. ${ }^{26,27}$

We sought to understand the baseline level knowledge of CMV in a women's health clinic in Minneapolis, MN and to both develop an effective mode of education to increase awareness of cCMV and to implement strategies to prevent CMV infection during pregnancy. We developed an educational handout that can be easily utilized during routine prenatal care to educate pregnant women about CMV infection and cCMV. After receiving the CMV education, the study participants were asked about their level of satisfaction with the education and perceived value of the information.

\section{Materials and Methods Study Design}

This prospective intervention study was performed from July 2018 through April 2019 at Women's Health Specialist Clinic, the clinic associated with the Department of Obstetrics, Gynecology and Women's Health at the University of Minnesota. The study was approved by the University of Minnesota Internal Review Board prior to starting enrollment (study identification number 00002735). Women with intrauterine pregnancies (IUP) at less than 34 weeks of estimated gestational age (EGA) were eligible for enrollment in the study. Additional inclusion criteria included: able to give informed consent, age greater than or equal to 18 years old, and receiving prenatal care at Women's Health Specialists clinic. Informed written consent was obtained prior to enrollment in the study. The consent forms were available in English, Spanish and Somali. An in-person interpreter was used for other languages. After informed consent was obtained, participants were given the pre-educational handout 
questionnaire (supplemental materials 1). The questionnaire was in English. If the patient's primary language was other than English, an in-person interpreter was available to assist them in filling out the questionnaire. After the participant had completed the pre-educational questionnaire, she was given the educational handout about CMV to take home (supplemental materials 2). This handout was designed by the study authors using references from the CDC, ${ }^{28}$ the Society for Maternal Fetal Medicine ${ }^{8}$ and the American College of Obstetricians and Gynecologists ${ }^{29}$ and was available in English, Spanish and Somali languages. The obstetrical provider conducting the prenatal visit did not provide education about $\mathrm{CMV}$ or review the educational handout with the study participant. At the next scheduled prenatal clinic visit, the post-educational handout questionnaire was administered (supplemental Figure 3) which was 2-4 weeks after the handout was given. This questionnaire included questions regarding their level of satisfaction with the education and perceived value of the information. All consent forms, questionnaires and the educational handout were provided on paper. The study participants did not receive any gifts or monetary compensation for participating in the study.

\section{Statistical Analysis}

Descriptive statistics were summarized as frequencies and percentages for categorical measures, and numeric measures were summarized with means and standard deviations. For the subjects who completed both surveys, McNemar's test was used to test whether proportions of subjects who heard of the congenital conditions changed between the pre- and post-handout survey. R (Version 3.5.0) was used for statistical analyses. P-values less than 0.05 were considered statistically significant.

\section{Results}

This study was conducted in a large academic obstetrical practice in Minneapolis, MN. The enrollment period was July 2018-April 2019. A total of 263 women with IUP at $<34$ weeks of EGA were enrolled in the study, and 215 completed both questionnaires. Of the women to complete either of the questionnaires, some women only partially completed the questionnaires and those partial responses were recorded and included in the analysis. The results are presented as a percent and the number of women who answered each question is reported with the results. Of the 48 participants that did not complete the post-educational questionnaire: 42 declined to complete the post-educational questionnaire or did not return to clinic, 3 delivered before they were able to complete post-educational questionnaire, 2 had a spontaneous abortion, 1 terminated the pregnancy. The baseline characteristics of the study participants are reported in Table 1. Some participants declined to answer all the baseline demographic questions. The mean age was 31.9 years of age. The majority of the population identified was white (68.2\%, [176/258]) and were born in the United States (79\% [209/263]). A large portion reported having a college degree or more $(40.3 \%$ [106/263] college graduate and $38.4 \%$ [101/263] post-college graduate). Just under half (45.5\% [116/255]) reported a child at home. Of the participants who reported having a child, $48.4 \%(56 / 116)$ reported a child in daycare. A small fraction (10.1\% [25/248]) reported ever having a job in a childcare facility, and $32.9 \%(83 / 252)$ reported having a job in healthcare.

During routine prenatal clinic visits, study participants were given an educational handout that included information

Table I Baseline Characteristics of Study Participants

\begin{tabular}{|l|l|}
\hline $\begin{array}{l}\text { Age } \\
\text { Mean (SD) } \\
\text { Range }\end{array}$ & $31.9(4.6)$ \\
\hline Ethnicity & $18.0-44.0$ \\
\hline White & \\
Asian & $176(68.2 \%)$ \\
Black & $24(9.3 \%)$ \\
Hispanic & $21(8.1 \%)$ \\
Other & $17(6.6 \%)$ \\
Somali & $11(4.3 \%)$ \\
\hline Birth County & $9(3.5 \%)$ \\
United States & \\
Other & $209(79.5 \%)$ \\
\hline Education & $54(20.5 \%)$ \\
Some high school & \\
High school graduate & $9(3.4 \%)$ \\
Associate degree/some college & $15(5.7 \%)$ \\
College graduate & $32(12.2 \%)$ \\
Post-college degree & $106(40.3 \%)$ \\
\hline Child at home & $101(38.4 \%)$ \\
\hline Yes & \\
No & $116(45.5 \%)$ \\
\hline Job in Child Daycare & $139(54.5 \%)$ \\
Yes & $25(10.1 \%)$ \\
No & $223(89.9 \%)$ \\
\hline No in Healthcare & $83(32.9 \%)$ \\
\hline
\end{tabular}


about CMV infection, cCMV, and recommended increased hygiene practices (see Box 1 for a summary of the recommended hygiene practices; supplemental materials 2) designed to prevent maternal CMV infection. Prior to receiving CMV education, the participants were asked if they had heard of cCMV and other congenital conditions. A minority of participants had heard of CMV (33\%, [85/ 261]) and parvovirus B19 (30\%, [78/261]), whereas the majority of participants had heard of group B Streptococcus infection, rubella, spina bifida, fetal alcohol syndrome, Zika virus infection, syphilis, Down syndrome and HIV/AIDS. Almost half (48\%, [126/261]) had heard of Toxoplasma infection (Figure 1). A post-educational handout questionnaire was administered at the next scheduled prenatal visit. The majority, $73 \%$ (157/215), of participants indicated that they remembered reading the educational handout. The number of participants who had heard of CMV increased significantly (36\% [77/214] to $75 \%$ [160/214], p-value $<0.001$, $\mathrm{N}=214$, Figure 2). The statistically significant increase in the number of participants who indicated that they had heard of CMV indicated an increase in CMV knowledge. The number of participants who had heard of parvovirus B19 $(32 \%$ [69/213] to $46 \%$ [97/213], p-value <0.001) and
Toxoplasma (50\% [107/214] to 57\% [122/214], p-value $0.005)$ also increased but to a lesser degree. The number of participants who had heard of rubella, spina bifida, fetal alcohol syndrome, Zika virus, syphilis, Down syndrome and HIV/AIDS did not change significantly.

After receiving education about increased hygiene practices to prevent CMV infection, the participants were asked how they felt about the recommendations. Participants were asked to rank each recommendation on a scale of 1 to 5 . A rank of 1 indicated "I felt the recommendation was very difficult to do and I could not do it", a rank of 3 indicated "I felt neutral about the recommendation" and a rank of 5 indicated "I felt the recommendation was easy to do and I was able to do it". The rank for each recommendation was averaged (Figure 3). Overall, the increased hygiene practices were well-received. The recommendations to "wash your hands after changing a diaper" and "do not share a toothbrush with your child" received the highest average scores (4.7, standard deviation 0.60 and 0.67 , respectively) and the recommendations to "wash hands after handling children's toys" and "do not kiss your child on the mouth" received the lowest average scores (3.8 and 4.0, respectively). Participants were asked if they had tried to

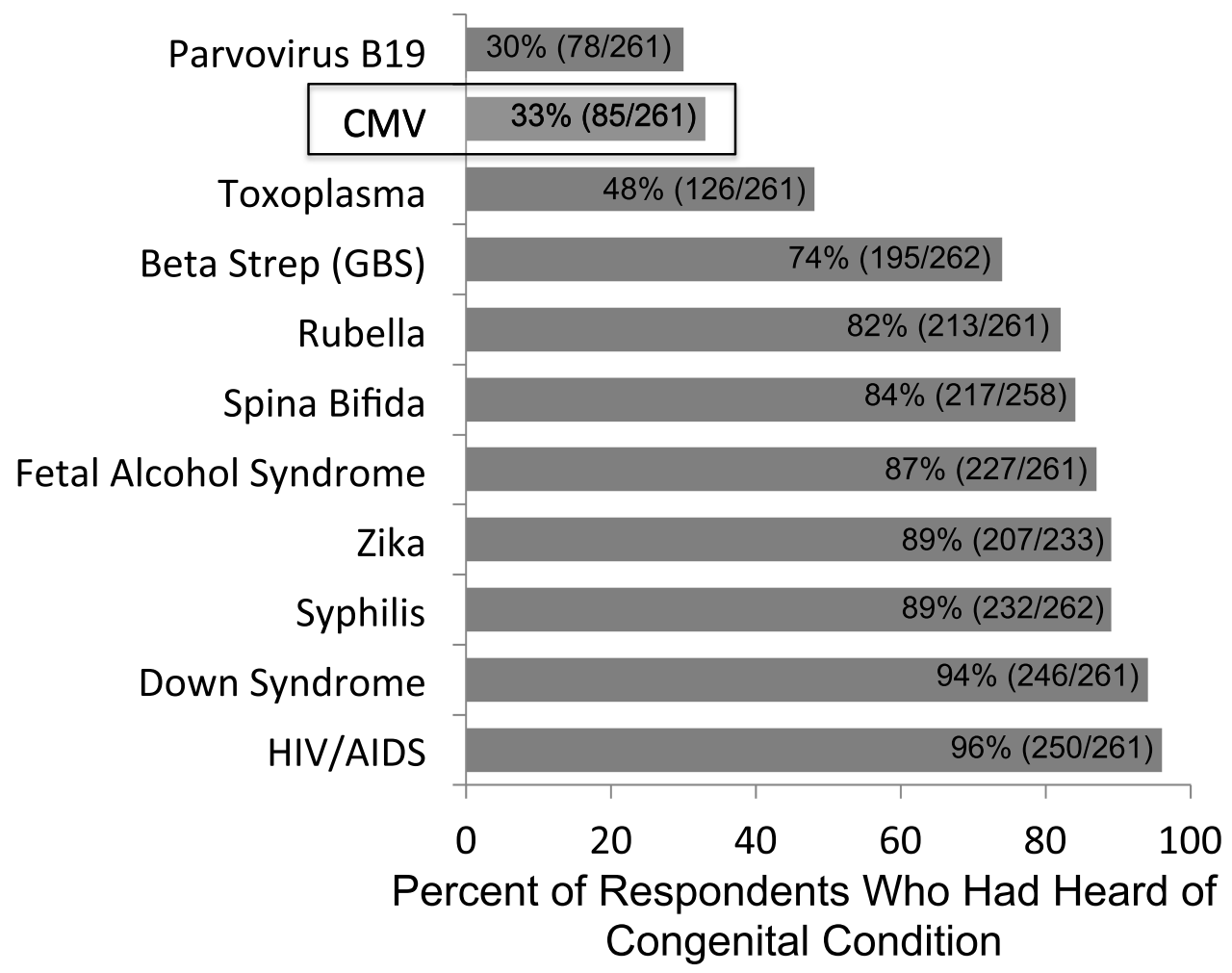

Figure I Baseline knowledge of CMV is low compared to other congenital conditions. Respondents were asked to respond either yes or no if they had heard of each congenital condition. The percent that responded "yes" is shown. 


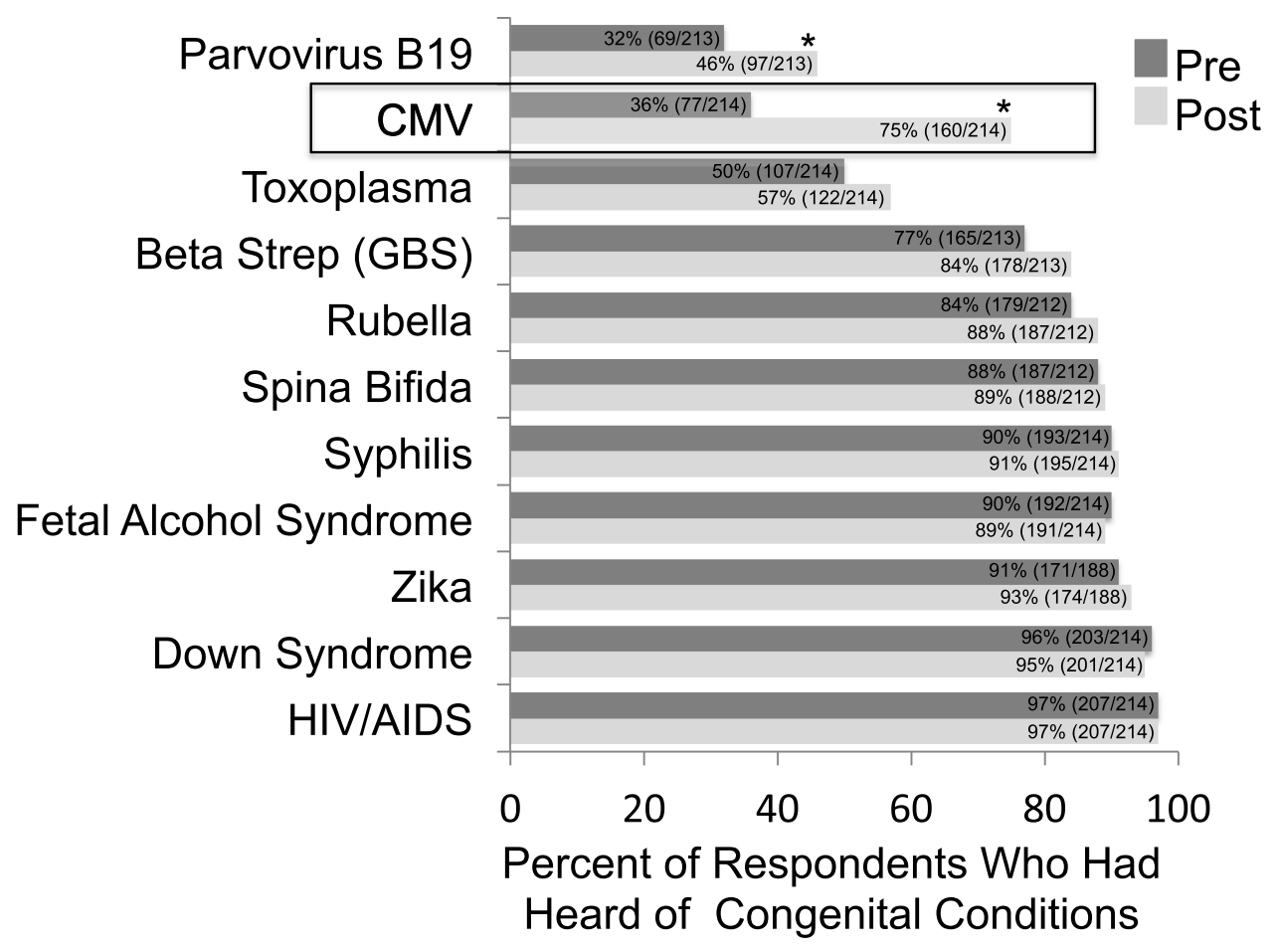

Figure 2 Education about CMV infection provided during prenatal clinic visits increased knowledge of CMV infection is shown. Percent of respondents that indicated they had heard of CMV compared to other congenital infections before and after CMV education provided during prenatal clinic visits. The CMV educational handout was designed by the study authors and was given to respondents after they had completed the pre-handout questionnaire. *p-value $<0.00$ I, McNamar's test.

implement the recommendations at home and 74\% (134/ 180) of participants responded that they had tried to increase their hand hygiene after receiving CMV education. Figure 4 demonstrates how participants reported hand hygiene practices before and after receiving education about CMV and the increased hand hygiene practices to prevent CMV infection that were undertaken. Participants were asked to rank on a scale of 1-5 how often they performed the hand hygiene practice at home. A rank of 1 indicated "I do not do that", a rank of 3 indicated "I do that half of the time" and a rank of 5 indicated "I do that all the time". The rank for each recommendation from the respondents was averaged and an average score was generated. There was a statistically significant increase in participants that performed hand hygiene after wiping a child's nose or drool (average score 3.5 increased to 3.9, $\mathrm{p}=0.01$ ) and handling children's toys (average score 2.4 increased to $3.1, \mathrm{p}=<0.001)$. There was also a statistically significant increase in the number of participants that also avoided saliva when kissing children (average score 3.4 increased to $3.9, \mathrm{p}=0.007$ ). There was not a significant difference in hand hygiene practices after changing a diaper (average score 4.4 increased to $4.9, \mathrm{p}=$ not significant [NS]), feeding young children (average 3.5 increased to $3.8, \mathrm{p}=\mathrm{NS}$ ), sharing a toothbrush (average score 1.2 to $1.3, \mathrm{p}=\mathrm{NS}$ ), cleaning surfaces that come into contact with a child's urine or saliva (average score 3.9 increased to $4.0, p=N S$ ) or kissing a child on the mouth (average score 2.8 decreased to $2.5, \mathrm{p}=\mathrm{NS}$ ).

The study participants were also asked about their level of satisfaction with the education and perceived value of the information. Nearly all the responding participants thought the handout was easy to $\operatorname{read}(95 \%, 181 / 191)$, were glad they read the handout $(96 \%, 180 / 188)$, and indicated that it provided useful information (98\%, 187/ 190) (Figure 5). Additionally, 98\% (187/190) felt more women should receive education about CMV infection during pregnancy.

\section{Discussion and Conclusion}

Even though CMV is a major source of neonatal morbidity, the majority of pregnant women have never heard of this infection. ${ }^{5-8}$ While prevention of congenital CMV is an important public health goal, there is not an approved CMV vaccine, nor are there medical therapies to prevent or treat in utero infection. Currently, the only recognized intervention to decrease cCMV is increased hygiene practice. Previous studies have demonstrated that counseling 


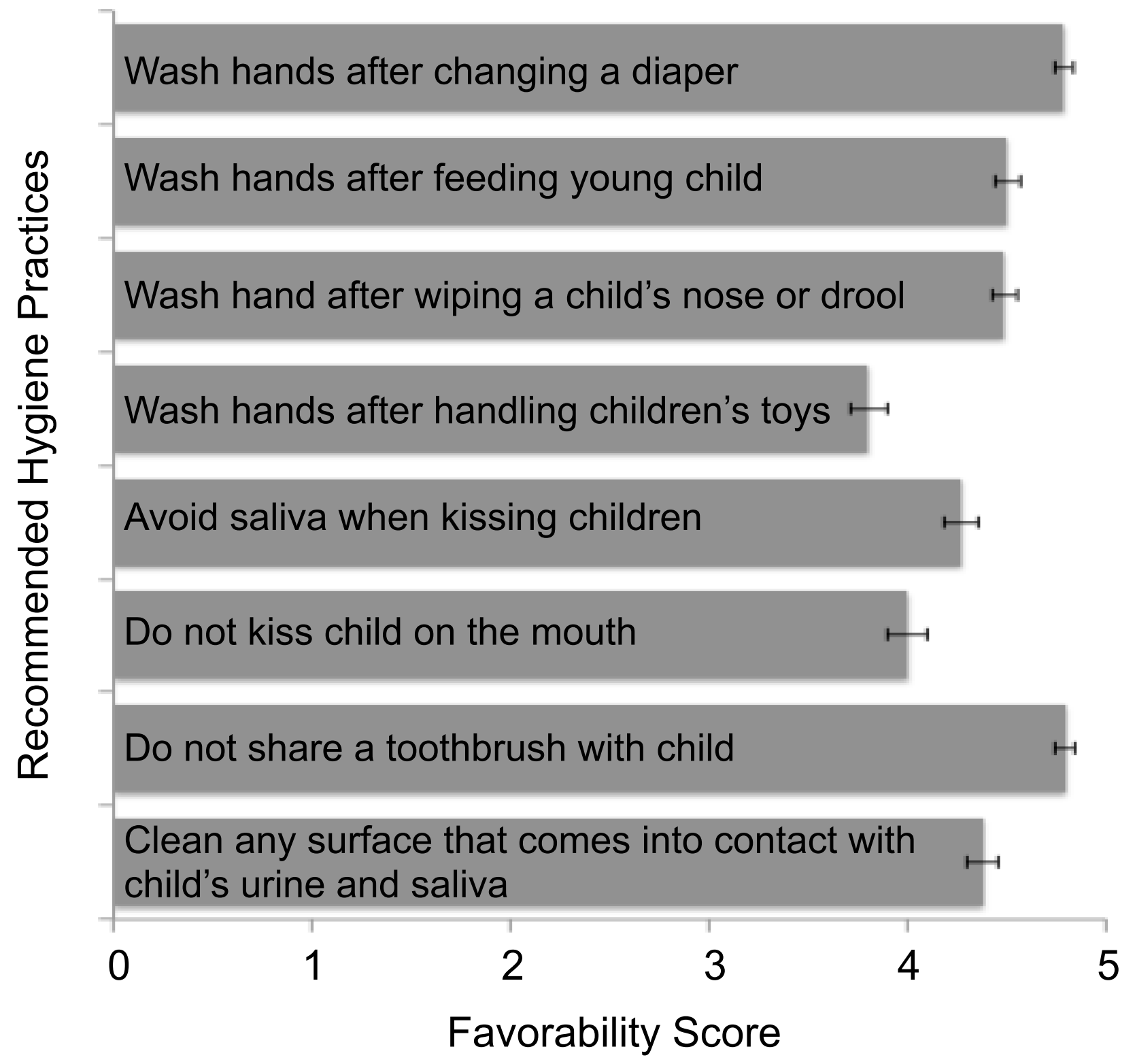

Figure 3 The recommendations for increased hygiene practices were well-received by respondents. Respondents were asked to rank how they felt about each hygiene recommendation on a scale of I-5. A rank of I indicated "I felt the recommendation was very difficult to do and I could not do it", a rank of 3 indicated "I felt neutral about the recommendation" and a rank of 5 indicated "I felt the recommendation was easy to do and I was able to do it". The rank for each recommendation from the respondents was averaged and a favorability score was generated.

women about CMV infection and teaching hygiene practices can reduce the rate of maternal CMV infection. ${ }^{21-24}$ Despite this, education about cCMV and preventing maternal infection is not routinely discussed during prenatal care. $^{9-11}$ Our objective was to determine the baseline awareness of cCMV in the OB/GYN clinic associated with the University of Minnesota and design an educational tool that can be easily utilized in a busy obstetrical practice, emphasizing the role of hygienic practices in preventing infection.
We devised a prospective intervention study that allowed us to enroll a large number of participants and collect longitudinal data regarding awareness of CMV infection and openness to CMV education. Our data demonstrated a low baseline awareness of maternal and cCMV, consistent with previously published studies. ${ }^{6-8}$ After receiving the CMV educational handout, the majority of study participants reported they remembered reading the educational handout and the awareness of CMV increased significantly. When the participants were asked 


\section{Wash hands after changing a diaper}

Wash hands after feeding young child

Wash hand after wiping a child's nose or drool

\section{Wash hands after handling children's toys}

\author{
Avoid saliva when \\ kissing children \\ Do not kiss child \\ on the mouth \\ Do not share a \\ toothbrush with child \\ Clean any surface that \\ comes into contact with \\ child's urine and saliva
}

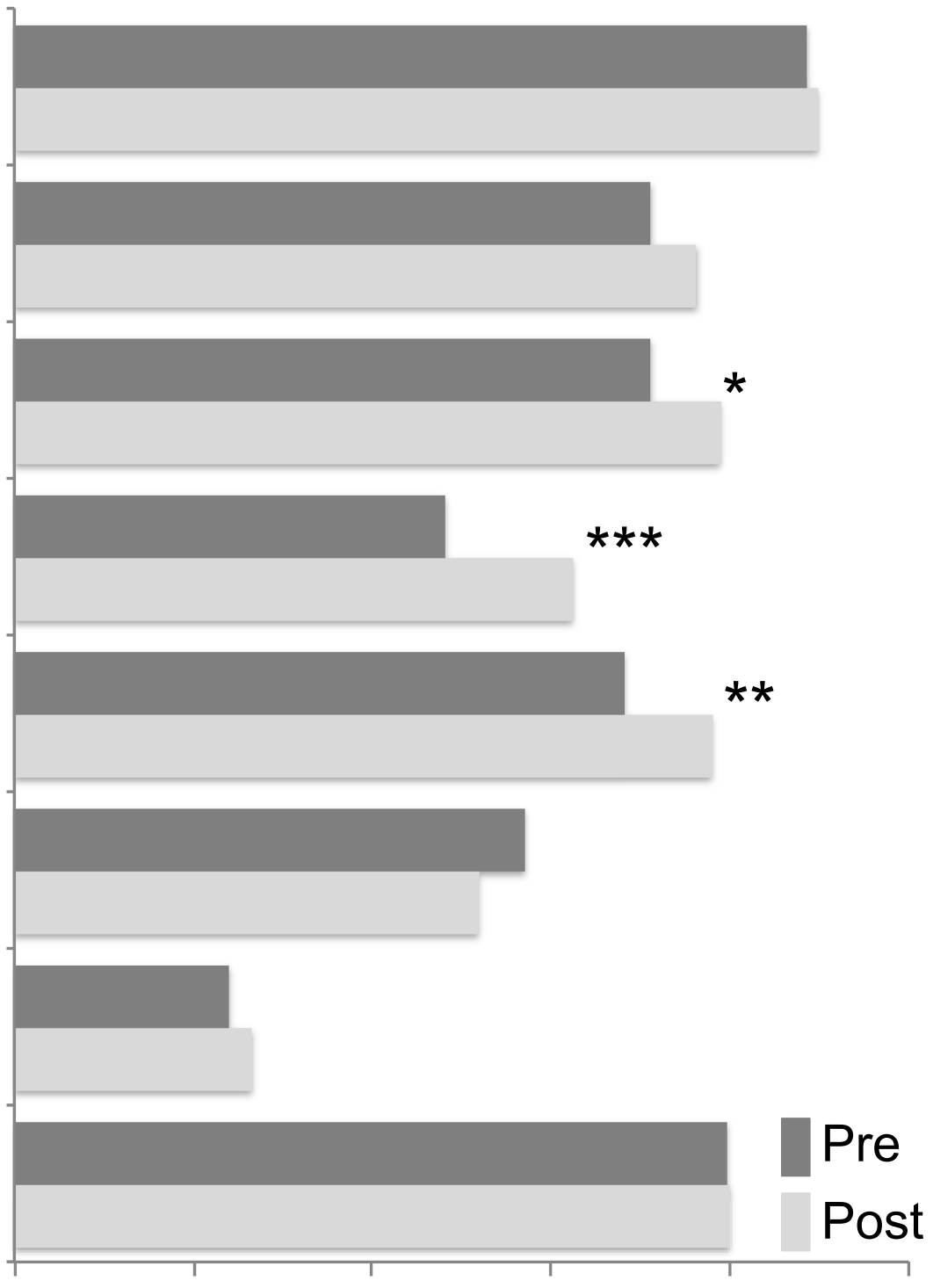

\section{Average Score}

Figure 4 Respondents increased hand hygiene practices after CMV education. Respondents were asked to rank on a scale of I-5 how often they do hand hygiene at home. A rank of I indicated "I do not do that", a rank of 3 indicated "I do that half of the time" and a rank of 5 indicated "I do that all the time". The rank for each recommendation from the respondents was averaged and an average score was generated. ${ }^{*} \mathrm{p}=0.0 \mathrm{I},{ }^{* *} \mathrm{p}=0.007,{ }^{* * *} \mathrm{p}=<0.00 \mathrm{I}$, Paired $t$-test.

their opinion of the CMV education and hygiene recommendations, receipt of the information was viewed positively and the majority of study participants reported they implemented the recommended hygiene practices at home. Specifically, our data show that participants increased hand hygiene after wiping a child's nose or drool and after handing children's toys and participants avoided saliva when kissing children. Prior to CMV and hand hygiene education, participants reported they were already practicing hand hygiene after changing a diaper, feeding young children, cleaning surfaces that come into contact with urine and saliva and not sharing a toothbrush with a child; therefore, it is not unexpected that there was not a statistically significant change in these hand hygiene practices. The exception to this is the recommendation of "do not kiss child on the mouth", which participants ranked as only doing this recommendation half of the time prior to education. The rank did not change after 


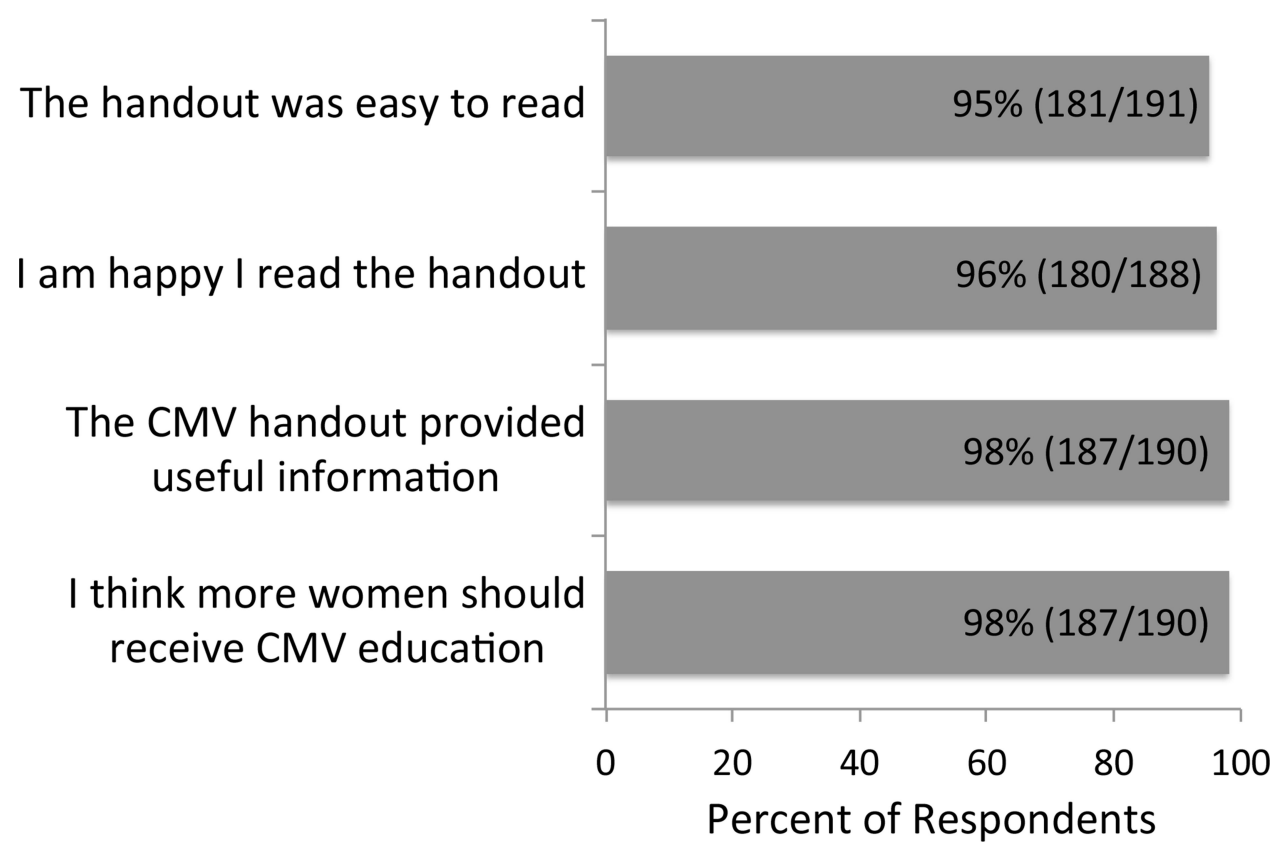

Figure $5 \mathrm{CMV}$ education was well received by the respondents. The respondents were asked to respond either yes or no to questions regarding how they viewed the CMV education. These questions were asked on the post-handout questionnaire.

education. While we cannot know for certain why the participants did not implement this recommendation, it could be speculated that kissing that child on the mouth is a sign of affection and the need to express affection outweighed the risk of CMV. Most participants thought the CMV educational handout was easy to read, provided useful information and thought more women should receive this education during prenatal care. In conclusion, the baseline awareness of CMV among pregnant women is low but can be significantly increased with CMV education provided during routine prenatal care and women are willing to implement increased hygiene practices to prevent CMV infection.

Since this study was conducted during routine prenatal care visits in a busy obstetrical practice using a simple, paperbased educational handout, our findings are applicable to most obstetrical practices and can be easily integrated into prenatal clinical practice. When these findings are combined with previous studies showing decreased maternal CMV infection after counseling about increased hygiene practices, there is a strong case to incorporate CMV education into routine prenatal care. Integrating CMV education into routine prenatal care is especially important in obstetrical clinics that provide care to racial and ethnic minority women since these populations have an increased prevalence of cCMV compared to non-Hispanic white populations. ${ }^{30}$ The simple, low-tech method of CMV education included in this study provides an easy, readily available resource that can be utilized by most obstetrical practices.

However, there are limitations to this study. This study was conducted at an academic clinic practice that cares for a population of women that is predominantly white (68.2\%). Because of this demographic, this study may not be generalizable to the overall population or clinics with predominantly minority or immigrant populations. Additionally, our study population was also well educated with $40.3 \%$ of participants reporting a college degree and $38.4 \%$ reporting a postcollege degree and this may not be representative of most clinic patient populations. However, it should be noted that our study population also had a significant portion of participants with less education and these patients also benefited from CMV education. Given the educational diversity of our study population and the high acceptance of hand hygiene, we propose educational background is not a barrier to the use of a paper handout to provide CMV education, and that use of such handouts can lead to increased hygienic practices that can prevent $\mathrm{CMV}$ infection. An additional limitation to this study may be response bias. In response to CMV education, the percent of participants who had heard of CMV increased but the number of participants who had heard of Parvovirus B19 and Toxoplasma also demonstrated a statistically significant increase. This suggests that some of the increase in CMV awareness could be due to response bias. While response bias may have contributed, there are additional 
explanations to consider that could explain the increase in awareness of Parvovirus B19 and Toxoplasma, such as increased independent research of congenital conditions after learning about cCMV. Despite these limitations, our data support the conclusion that an educational handout about cCMV and CMV infection can lead to increased hygiene practice, and is an effective method to educate pregnant women about CMV during prenatal care.

In order to incorporate CMV education into routine prenatal care, we need to understand the barriers to providing this education. Potential barriers include limited time during prenatal care visits, low CMV knowledge among obstetrical providers, a lacking of understanding about the significance of cCMV, ${ }^{9-11,31,32}$ a lack of CMV educational resources and the misconception that there is no way to prevent cCMV. This misconception can lead clinicians to wrongly assume that they should not bother patients with education about cCMV, since there is nothing that can be done to prevent the infection. Future directions should aim to understand barriers to CMV education and develop strategies to overcome them.

In summary, this study demonstrates that education about cCMV, maternal CMV infection and increased hygiene practices given during routine prenatal care can increase awareness among pregnant women. Pregnant women view this education favorably, are willing to implement increased hygiene practices to prevent maternal CMV infection, and think this education should be part of routine prenatal care. We propose that obstetrical providers should incorporate $\mathrm{CMV}$ education into their routine prenatal care.

\section{Acknowledgments}

We would like to thank all the women who participated in this study. Research was approved by the University of Minnesota Internal Review Board study identification number 00002735. Research reported in this publication was supported by NIH grant P30CA077598 utilizing the Biostatistics and Bioinformatics Core shared resource of the Masonic Cancer Center, University of Minnesota and by the National Center for Advancing Translational Sciences of the National Institutes of Health Award Number UL1-TR002494. The content is solely the responsibility of the authors and does not necessarily represent the official views of the National Institutes of Health. Grant support: R01 R01 HD098866 (MRS). This work was also supported by the University of South Carolina's Disability Research and Dissemination Center (DRDC) through its Cooperative Agreement (Number 6U19DD001218) with the Centers for Disease Control and Prevention (CDC). The Jamie McNew
Educational Endowment fund for CMV research is gratefully acknowledged (http://cmv.umn.edu/mcnew-lecture). These data were presented in abstract/poster format at The Pregnancy Meeting of the Society for Maternal Fetal Medicine in February 6, 2020, in Grapevine, TX and at the University of Minnesota Department of Pediatrics "PRESS" Conference, Masonic Children's Hospital, Minneapolis, MN, April 24, 2020.

\section{Disclosure}

Mark Schleiss reports personal fees from Moderna Vaccines and grants from NIH during the conduct of the study and advises this work was also supported by the University of South Carolina's Disability Research and Dissemination Center (DRDC) through its Cooperative Agreement (Number 6U19DD001218) with the Centers for Disease Control and Prevention (CDC). The authors report no other potential conflicts of interest in this work.

\section{References}

1. Cheeran MC-J, Lokensgard JR, Schleiss MR. Neuropathogenesis of congenital cytomegalovirus infection: disease mechanisms and prospects for intervention. Clin Microbiol Rev. 2009;22(1):99-126. doi:10.1128/CMR.00023-08

2. Schleiss MR. Congenital cytomegalovirus: impact on child health. Contemp Pediatr. 2018;35(7):16-24.

3. Cannon MJ. Congenital cytomegalovirus (CMV) epidemiology and awareness. J Clin Virol. 2009;46((SUPPL. 4)). doi:10.1016/j. jev.2009.09.002

4. Cannon MJ, Davis KF. Washing our hands of the congenital cytomegalovirus disease epidemic. BMC Public Health. 2005;5:1-8. doi:10.1186/1471-2458-5-70

5. Jeon J, Victor M, Adler SP, et al. Knowledge and awareness of congenital cytomegalovirus among women. Infect Dis Obstet Gynecol. 2006;2006. doi:10.1155/IDOG/2006/80383

6. Tastad KJ, Schleiss MR, Lammert SM, Basta NE. Awareness of congenital cytomegalovirus and acceptance of maternal and newborn screening. PLoS One. 2019;14(8):e0221725. doi:10.1371/journal. pone. 0221725

7. Cannon MJ, Westbrook K, Levis D, Schleiss MR, Thackeray R, Pass RF. Awareness of and behaviors related to child-to-mother transmission of cytomegalovirus. Prev Med. 2012;54(5):351-357. doi:10.1016/j.ypmed.2012.03.009

8. Hughes BL, Gyamfi-Bannerman C. Diagnosis and antenatal management of congenital cytomegalovirus infection. Am J Obstet Gynecol. 2016;214(6):B5-B11. doi:10.1016/j.ajog.2016.02.042

9. Centers for Disease Control and Prevention.Knowledge and practices of obstetricians and gynecologists regarding cytomegalovirus infection during pregnancy-United States, 2007. MMWR Morb Mortal Wkly Rep. 2008;57(3):65-68.

10. Ross DS, Rasmussen SA, Cannon MJ, et al. Obstetrician/ Gynecologists' knowledge, attitudes, and practices regarding prevention of infections in pregnancy. $J$ Women's Heal. 2009;18 (8):1187-1193. doi:10.1089/jwh.2008.1288

11. Pereboom MTR, Manniën J, Spelten ER, Hutton EK, Schellevis FG. Maternal cytomegalovirus infection prevention: the role of Dutch primary care midwives. Midwifery. 2014;30(12):1196-1201. doi:10.1016/j.midw.2014.04.007 
12. Dollard SC, Grosse SD, Ross DS. New estimates of the prevalence of neurological and sensory sequelae and mortality associated with congenital cytomegalovirus infection. Rev Med Virol. 2007;17 (5):355-363. doi:10.1002/rmv.544

13. Kenneson A, Cannon MJ. Review and meta-analysis of the epidemiology of congenital cytomegalovirus (CMV) infection. Rev Med Virol. 2007;17(4):253-276. doi:10.1002/rmv.535

14. Permar SR, Schleiss MR, Plotkin SA. Advancing Our Understanding of Protective Maternal Immunity as a Guide for Development of vaccines to reduce congenital cytomegalovirus infections. J Virol. 2018;92:7. doi:10.1128/JVI.00030-18

15. Colugnati FAB, Staras SAS, Dollard SC, Cannon MJ. Incidence of cytomegalovirus infection among the general population and pregnant women in the United States. BMC Infect Dis. 2007;7. doi:10.1186/1471-2334-7-71.

16. Read JS, Cannon MJ, Stanberry LR, Schuval S. Prevention of mother-to-child transmission of viral infections. Curr Probl Pediatr Adolesc Health Care. 2008;38(9):274-297. doi:10.1016/j. cppeds.2008.08.001

17. Adler SP. Molecular epidemiology of cytomegalovirus: a study of factors affecting transmission among children at three day-care centers. Pediatr Infect Dis J. 1991;10(8):584-590. doi:10.1097/ 00006454-199108000-00007

18. Adler SP. Cytomegalovirus and child day care. $N$ Engl J Med. 1989;321(19):1290-1296. doi:10.1056/NEJM198911093211903

19. Ross DS, Victor M, Sumartojo E, Cannon MJ. Women's knowledge of congenital cytomegalovirus: results from the 2005 HealthStyles $^{\mathrm{TM}}$ survey. J Women's Heal. 2008;17(5):849-858. doi:10.1089/jwh. 2007.0523

20. Thackeray R, Magnusson BM. Women's attitudes toward practicing cytomegalovirus prevention behaviors. Prev Med Reports. 2016;4:517-524. doi:10.1016/j.pmedr.2016.09.008

21. Revello MG, Tibaldi C, Masuelli G, et al. Prevention of primary cytomegalovirus infection in pregnancy. $E$ Bio Med. 2015;2 (9):1205-1210. doi:10.1016/j.ebiom.2015.08.003

22. Adler SP, Finney JW, Manganello AM, Best AM. Prevention of child-to-mother transmission of cytomegalovirus among pregnant women. J Pediatr. 2004;145(4):485-491. doi:10.1016/j.jpeds.2004. 05.041
23. Adler SP, Finney JW, Manganello AM, Best AM. Prevention of child-to-mother transmission of cytomegalovirus by changing behaviors: a randomized controlled trial. Pediatr Infect Dis J. 1996;15 (3):240-246. doi:10.1097/00006454-199603000-00013

24. Vauloup-Fellous C, Picone O, Cordier AG, et al. Does hygiene counseling have an impact on the rate of CMV primary infection during pregnancy? Results of a 3-year prospective study in a French hospital. $J$ Clin Virol. 2009;46((SUPPL. 4)). doi:10.1016/j. jcv.2009.09.003

25. Price SM, Bonilla E, Zador P, Levis DM, Kilgo CL, Cannon MJ. Educating women about congenital cytomegalovirus: assessment of health education materials through a web-based survey. $B M C$ Womens Health. 2014;14:1. doi:10.1186/s12905-014-0144-3

26. Rawlinson WD, Boppana SB, Fowler KB, et al. Congenital cytomegalovirus infection in pregnancy and the neonate: consensus recommendations for prevention, diagnosis, and therapy. Lancet Infect Dis. 2017;17(6):e177-e188. doi:10.1016/S1473-3099(17)30143-3

27. National CMV Foundation. Available from: https://www.natio nalcmv.org/about-us/advocacy. Accessed May 20, 2020

28. CDC Cytomegalovirus (CMV) and Congenital CMV infection. Available from: https://www.cdc.gov/cmv/congenital-infection.html. Accessed December 07, 2020.

29. Practice bulletin no. 151: cytomegalovirus, parvovirus B19, varicella zoster, and toxoplasmosis in pregnancy. Obstet Gynecol. 2015;125 (6):1510-1525. doi:10.1097/01.AOG.0000466430.19823.53.

30. Fowler KB, Ross SA, Shimamura M, et al. Racial and ethnic differences in the prevalence of congenital cytomegalovirus infection. $J$ Pediatr. 2018;200:196-201.e1. doi:10.1016/j.jpeds.2018.04.043

31. Korver AMH, de Vries JJC, de Jong JW, Dekker FW, Vossen ACTM, Oudesluys-Murphy AM. Awareness of congenital cytomegalovirus among doctors in the Netherlands. J Clin Virol. 2009;46:S11-S15. doi:10.1016/j.jcv.2009.09.006

32. Cordier AG, Guitton S, Vauloup-Fellous C, et al. Awareness of cytomegalovirus infection among pregnant women in France. J Clin Virol. 2012;53(4):332-337. doi:10.1016/j.jcv.2011.12.031

\section{Publish your work in this journal}

The International Journal of Women's Health is an international, peerreviewed open-access journal publishing original research, reports, editorials, reviews and commentaries on all aspects of women's healthcare including gynecology, obstetrics, and breast cancer. The manuscript management system is completely online and includes a very quick and fair peer-review system, which is all easy to use. Visit http://www.dovepress.com/testimonials.php to read real quotes from published authors. 